merey, knowledge of birth control must be made available to all the peoples of the world."

Dr. R. Passmore provides what was for the oceasion an adequate survey of nutritional needs, quantitative and qualitative. $\mathrm{He}$ is particularly valuable in his tables and commentaries on obesity and relative 'under-weight' - the two complaints that to-day mark the contrasts between different peoples and, indeed, between different classes of any one country. Mr. N. W. Pirie was appropriately chosen to deal at the symposium with one of the subjects he has made his own, the scientific problems associated with future sources of food. As might be expected, he finds many new variations to play on the theme, while not neglecting the familiar approaches to it. Speaking of crops that are the raw material of biochemical engineering, he comments: "Enthusiasm for algal culture as an alternative to agriculture is now declining. ... When higher plants and algae are compared in comparable conditions, they have comparable capabilities and the techniques of husbandry are very much simpler with the higher plants". It is incidentally very useful that he records some of the figures which show the advantage of 'culling' wild animals for food on unimproved land, as contrasted with the attempted use of that land for the grazing of domestic livestock.

The final paper by Prof. Colin Clark treats of the economic problems. His argument merits very close attention. $\mathrm{He}_{\mathrm{e}}$ examines, for example, the varying calorie requirements under varying temperature conditions. He estimates in wheat equivalents-a method of calculation that has to be followed with care. The figures of the world's cultivable land are on the whole well recognized; but they deserve constant repetition. Thus, "The Japanese live healthily on 0.56 tons wheat equivalent per person per year, which they produce on 0.07 hectares (one-sixth acre). To produce the same amount of food an Indian uses $3 \frac{1}{2}$ times as much land: in other words, Indian output could be increased $3 \frac{1}{2}$-fold if India could attain Japanese standards in the use of fertilizers, plant breeding and rural education".

Of this little volume in its entirety, one may say that, taken in conjunction with, for example, Possibilities of Increasing World Food Production (Rome: Food and Agriculture Organization, 1963), it supplies any competent teacher or popular lecturer with most of the basic materials he would need; the conversion of detailed statistics might safely be left to those who know what their classes or audiences can assimilate. Whatever may happen to human numbers, the goal of doubling or trebling food production is certainly within our present power; and it should surely be as capable of stimulating the imagination of the young as any space exploration.

$$
\text { F. Le Gros Ciark }
$$

\section{A RUSSIAN VIEW OF NUCLEAR PHYSICS}

\section{Contemporary Models of the Atomic Nucleus}

By P. E. Nemirovskii. Translated from the Russian by S. and M. Nikolić. Translation edited by Prof. Bernard T. Feld. Pp. xii + 332. (London and New York : Pergamon Press, 1963.) 100s. net.

\section{Nuclear Models}

By Dr. P. E. Nemirovskii. Translated by S. Chomet. Pp. $x+372$. (London : E. and F. N. Spon, Ltd., 1963.) 70s. net.

$\mathrm{O}$ CE again we are confronted with two translations from the Russian of a single book on nuclear physics. We, the present reviewers, are not qualified to judge the publishing niceties of this practice-the question of 'authorization' of a translation, for example-but one does not have to ponder long on the considerable initial expense and effort involved in translating a book such as this and setting it up in print to realize that this sort of duplication is a singularly wasteful form of competition.

The book is basically a review of work done on various nuclear models up to the end of 1959. After an uninspiring start, in which the shell model is prosented as a long and dull compilation of facts, it improves somewhat to give a straightforward account of the collective model. The best sections, however, are those which discuss the optical model and related topies. These are based largely on the original work of the author and of other Russian physicists, and bring together much material otherwise scattered through the journals. The optical model formalism is presented more completely than in most text-books and there are quite extensive tables of sticking coefficients. The book then returns to the shell model and a discussion of the stripping reaction. The last chapter is devoted to radiative transitions and to $\alpha$-decay.

The theory of nuclear structure is now moving rapidly once more, and this book must be regarded as an introduction to these 'standard' nuclear models, rather than a review of the present-day situation. In this it is successful to a varying degree as we have indicated here; we must, however, record that we flatly disagree with the author's statement (Section 33) that intermediate coupling calculations for nuclei beyond mass 16 are either "not very significant" (Spon) or "of no use" (Pergamon).

Each translation has occasional inaccuracies and infelicities; we much preferred the Pergamon version, which is also better printed. Against this, however, must be set its considerably higher price.

\section{ELTZABETH BRADFORD}

J. M. Soper

\section{KEEPING UP-TO-DATE WITH RADIO ASTRONOMY}

\section{Radio Astronomy To-day}

Papers, designed to present Radio-Astronomy in all its Aspects, given at the Jodrell Bank Summer School, 1962. Edited by H. P. Palmer, R. D. Davies and M. I. Large. Pp. viii $+242+4$ plates. (Manchester: The University Press, 1963.) 32s. $6 d$.

Tring at

HIS book is a written account of the lectures and discussions at a summer school in radio astronomy held at Jodrell Bank in 1962. The lecturers were drawn not only from the staff at Jodrell Bank, but also included several distinguished visitors from other British and overseas universities. The contributions consequently provide an authoritative account of the scope, the achievements and problems of radio-astronomical research.

The book deals with a considerable variety of topics, and although the description on the title page, "Papors designed to present Radio Astronomy in all its Aspects", may be an over-statement, most subjects of present-day interest in this field are included. Altogether there are twenty articles, starting with contributions on solar and planetary omissions, and then ranging over such themes as radar detection of planets, radio telescopes, radio observations of galaxies and of interstellar matter, the theory of radiation processes, and ending with papers on cosmology.

Most of the articles resemble the useful survey papers sometimes given at introductions to conference sessions. In these circumstances, the audience is assumed to have at least some acquaintance with the subject, but wishes to have a brief review of basic facts and progress. The papers vary in length from 7 to 23 pages, and the shorter papers tend to suffer from too brief treatment. For example, the first article on "Radio Emissions from the Sun" is an interesting short essay comprising 23 pages, but the papers on "Planetary Emissions", 8 pages, and "Inter- 